\title{
Protección de las conquistas de la salud pública cubana ante la crisis financiera y económica mundial
}

\section{Protection of the Cuban public health achievements in the face of the world economic and financial crisis}

\author{
Néstor Marimón Torres ${ }^{\mathrm{I}}$; Evelyn Martínez $\mathrm{Cruz}^{\mathrm{II}}$ \\ IMáster en Salud Pública. Especialista de II Grado en Administración de la Salud. \\ Relaciones Internacionales del Ministerio de Salud Pública. La Habana, Cuba. \\ ${ }^{\mathrm{II}}$ Máster en Salud Pública y Salud Internacional. Especialista de I Grado en Medicina \\ General Integral. Relaciones Internacionales del Ministerio de Salud Pública. La \\ Habana, Cuba.
}

\section{RESUMEN}

Objetivos Analizar la repercusión de la crisis financiera y económica mundial sobre el Sistema Nacional de Salud y la preparación necesaria para preservar y mantener sus conquistas y logros.

Métodos Investigación cualitativa descriptiva detipo documental. Se analizaron documentos y publicaciones relacionados con el tema.

Resultados La crisis financiera tiene serias afectaciones sobre la salud de las poblaciones, se demostró que la mejor vía para enfrentarlas es a través de sistemas de salud con base en la atención primaria de salud, más equitativos y solidarios; con apropiado abordaje de los determinantes sociales e inversión en la formación de recursos humanos con otros valores éticos y morales. El ejemplo de Cuba con su Sistema Único de Salud y el nuevo modelo de formación de médicos corroboraron esta afirmaciones.

Conclusiones Las crisis que vive el mundo repercuten en la salud, más aún en la económica financiera. Se requieren sistemas de salud con cobertura universal que permitan alcanzar las metas propuestas. La experiencia cubana de crisis anteriores demuestra que un país requiere de inversión social, para mejorar la salud y sus determinantes y enfrentar y mitigar los efectos que ellas producen. Se requiere de solidaridad internacional, compartir lecciones aprendidas y unidad para enfrentar las consecuencias. El bloqueo económico y comercial que el gobierno de EE.UU. ejerce sobre Cuba, refuerza los efectos negativos de la crisis económica global. 
Palabras clave: Crisis financiera, sistemas de salud, atención primaria de salud, determinantes sociales, recursos humanos, solidaridad, bloqueo económico ycomercial, equidad, Cuba.

\section{ABSTRACT}

Objectives To analyze the impact of the world economic and financial crisis on the national healthcare system, and the required preparedness for preserving (protecting) and keeping its achievements.

Methods Documentary-type descriptive qualitative research. Documents and publications related to this issue were reviewed.

Results The financial crisis has severe impact on the population's health. It was demonstrated that the best way to face these negative effects is to develop more equitable and solidarity primary care-based health systems with adequate approach to social determinants and suitable level of investment in the formation of human resources fitted with ethical and moral values. The unique healthcare system and the new model of medical formation in Cuba is an example that confirms the abovementioned statements.

Conclusions The world financial and economic crisis affects health; therefore, health systems with universal coverage are required to reach the suggested goals. The Cuban experience gained in previous crisis demonstrates that a country needs social investment to improve health and its determinants, and to face and mitigate the effects of such crisis. International solidarity is needed, sharing of learned lessons and unity of actions to face the consequences are required. The economic and commercial blockade imposed on Cuba by the US government reinforces the negative effects of the global economic crisis.

Key words: Economic crisis, health systems, primary health care, social determinants, human resources, solidarity, economic and commercial blockade, equity, Cuba.

\section{INTRODUCCIÓN}

La salud en los últimos años se ha visto favorecida por políticas globales, regionales y locales; muchos países de ingresos bajos han aumentado el gasto sanitario. Además la ayuda destinada al sector se duplicó entre los años 2002 y $2006 .^{1}$

Se logró reducir la pobreza y mejorar los índices de salud, a través del cumplimiento de objetivos específicos como los Objetivos de Desarrollo del Milenio. Sin lugar a dudas, en los últimos tres decenios se ha producido un aumento de la riqueza en el mundo. En general, la población ha disfrutado de una vida más larga y sana. Pero esto esconde una realidad brutal: hoy las diferencias respecto a niveles de ingresos, las oportunidades y el estado de salud, tanto de los países como entre ellos, son mayores que en cualquier otro momento de la historia, afirmó la Directora General de la OMS, Dra. Margaret Chan en la 62 Asamblea Mundial. $^{2}$ 
Las desigualdades en los resultados de salud, en el acceso a la atención médica, medicamentos y redes de seguridad social, son grandes preocupaciones para todos, la diferencia en la esperanza de vida entre países ricos y pobres excede los 40 años, los gastos gubernamentales anuales per cápita en la salud van desde 20 USD hasta 6 mil.

A pesar del amplio arsenal con que cuenta la medicina hoy, casi 10 millones de niños de corta edad y mujeres embarazadas mueren prematuramente cada año por causa en gran medida evitables.

La OMS calcula que cada año los costos de la atención de salud arrastran alrededor de 100 millones de personas por debajo del umbral de pobreza, algo irónico, cuando el compromiso es reducir estos costos, y más amargo, en una época de crisis financiera. ${ }^{3}$

Se vaticina que en los países prósperos las personas perderán sus trabajos, sus hogares, sus ahorros y esto es trágico. En los países en desarrollo, se perderá la vida.

Hoy el mundo está inmerso en crisis, ya sea energética, alimentaria, climática o financiera, e incluso ante una posible crisis sanitaria por la pandemia de influenza $A$ H1N1. Todas afectan a casi la totalidad de los países con independencia del nivel de su desarrollo económico, es una grave y actual realidad.

Aunque es una crisis global, las consecuencias no se sentirán equitativamente. Serán más impactados los países en desarrollo y las poblaciones vulnerables, como sucede en todo tipo de crisis, no obstante, al afectarse y debilitarse todo el mundo, también se reduce el nivel de ayuda que reciben estos países.

La interconexión entre los mercados financieros, la economía y las empresas es mayor ahora que nunca, por lo que los efectos de la crisis pasan muy velozmente de unos países a otros y de un sector económico a otro. ${ }^{4}$

Cuba, con un modelo de desarrollo de carácter socialista, en el que la salud es un derecho de todos los ciudadanos y una responsabilidad del Estado y que posee un sólido Sistema de Salud, ya enfrentó en la década de los años 1990 una fuerte crisis económica, recrudecida por el bloqueo económico y comercial del gobierno de Estados Unidos sobre el país. Las acciones realizadas en ese momento, constituyen lecciones aprendidas, que sin duda servirán para enfrentar las repercusiones de la actual crisis financiera y económica global y podrán servir de referencia para algunos países de la región.

En este trabajo se profundizará en la evolución de la crisis financiera, que si en sus inicios fue exclusiva del sector inmobiliario en Estados Unidos de Norteamérica, hoy afecta a todas las economías, sectores y países, llegando a ser ya una crisis económica global con serias repercusiones sociales para todos los países. Se profundizará en la experiencia cubana de crisis anteriores y sus fortalezas para enfrentarla.

\section{MÉTODOS}

Se realizó una investigación cualitativa descriptiva de tipo documental cuyo propósito fundamental es proporcionar elementos que permitan realizar un análisis 
del impacto de la crisis financiera mundial sobre el Sistema Nacional de Salud cubano y la preparación necesaria para preservar los logros alcanzados en el mismo.

Para el desarrollo de este trabajo, se hizo un análisis documental de los efectos de la crisis financiera sobre los sistemas de salud y en especial del cubano.

Las categorías de análisis a tener en cuenta.

. Políticas Públicas.

. Gasto Público.

- Seguimiento en salud.

Las fuentes de información a utilizar:

- Documentos publicados por Organismos Financieros Internacionales.

- Documentos publicados por Organismos Internacionales de Salud.

- Documentos publicados y no publicados presentados en reuniones, foros, simposios, tanto nacionales como internacionales, a los que se pudo acceder.

- Informe anual de Cuba sobre los efectos del bloqueo de Estados Unidos de América.

Se analizaron documentos específicos existentes en el país, relacionados con el objeto de estudio, las publicaciones relacionadas con el enfrentamiento de Cuba a otras crisis (Periodo Especial) y se revisó la experiencia cubana en el desarrollo y perfeccionamiento de la Atención Primaria de Salud, los determinantes sociales y la formación de recursos humanos con el desarrollo del nuevo programa para ello.

Además, se tuvieron en cuenta los resultados anteriores y las experiencias susceptibles de ser aplicadas o ajustadas a este hipotético escenario. Se realizaron entrevistas semiestructuradas al personal que se consideró necesario para completar la información obtenida y se revisaron todos los documentos e informes relacionados con el impacto del bloqueo en la economía y sociedad cubana.

Los resultados en su mayoría fueron sometidos al análisis de contenido correspondiente y se presentan en relación al tipo de estudio, por lo que en su mayoría aparecerán en forma discursiva según las categorías seleccionadas para el análisis.

\section{RESULTADOS Y DISCUSIÓN}

\section{Crisis financiera global. Su desarrollo en el tiempo}

Se plantea que la actual crisis económica y financiera mundial es la peor que vive el mundo desde los años de 1930 y según algunos autores, va más allá al 
considerarla como una crisis estructural del sistema neoliberal, por lo que las soluciones que se plantean no son puramente económicas. ${ }^{5,6}$

Se han realizado pronósticos, que al paso del tiempo se han modificado, siendo cada vez más preocupantes. Primero, desaceleración del crecimiento en los países más industrializados y de un crecimiento de 6,4 \% esperado, sólo se alcanza el 4,5 en el 2009. El Banco Mundial estimaba que hasta ese momento, se habían afectado 94 de los 116 países en desarrollo y que 43 de ellos experimentan altos niveles de pobreza que se incrementaría de forma general a partir fundamentalmente de las pérdidas de empleo, disminuciones de salario y remesas, entre otros.

Posteriormente los reportes afirmaron que la economía mundial retrocederá aún más, el Fondo Monetario Internacional (FMI) estimaba al principio que el crecimiento económico para el 2009 sería de 2,2 \%, después de 0,5\% y finalmente, que seria negativo. El 27 de Mayo la ONU emitió un informe semestral del Departamento de Asuntos Económicos y Sociales (DESA) Ilamado Situación Económica Mundial y Perspectivas 2009, donde señala que la reducción será de 2, 6 $\%$ y la cifra de personas que incrementaran la pobreza ascenderá a 105 millones. ${ }^{7,8}$

Según cálculos de la Organización Internacional del Trabajo (OIT) el número total de desocupados en el mundo será entre 210 y 239 millones de personas. Los pronósticos sobre la cifra de desempleados en América Latina son de un incremento entre 3 y 4 millones, a lo que hay que agregar el galopante crecimiento del subempleo, como indicó a BBC Mundo el director del Instituto de Estudios Laborales de la OIT:

...En muchos países ha aumentado el número de gente que está trabajando a tiempo parcial aunque quisiera trabajar a tiempo completo. En muchos casos se ha llegado a acuerdos para reducir el número de horas de trabajo a cambio de conservar el puesto laboral.

Por ejemplo, en Estados Unidos el desempleo alcanza cifras del 10,2 \% que representa cerca de 16 millones de personas y en España alcanza el $20 \%$, lo que habla a favor de la profundización de los efectos de la crisis a pesar de la anunciada recuperación financiera. ${ }^{9}$

En los países ricos los desempleados están protegidos de las penurias gracias a la seguridad social, lo que no es igual en países subdesarrollados donde no tener trabajo significa pasar hambre y miseria. La gente busca cualquier tipo de trabajo sin importar sus condiciones o tipo de remuneración. Por lo tanto, la crisis económica aparecerá no solo con aumento del desempleo, sino también con trabajadores pobres y vulnerables y con el aumento de la pobreza y de la extrema pobreza también crece la mala salud. La pérdidas en el desarrollo cognitivo, emocional y físico de los niños que crecen en estas condiciones son potencialmente enormes. ${ }^{10}$

Recientemente la FAO dio a conocer el pronóstico de una cifra record de hambrientos para el 2009 de 1020 millones, a decir de Jacques Diouf, su Director General "La silenciosa crisis del hambre, que supone un riesgo para la paz y seguridad mundial". Se está muy lejos de alcanzar uno de los Objetivos de Desarrollo del Milenio que es recortar a la mitad el numero de la gente que pasa hambre en el mundo, a menos de 420 millones para el $2015 .^{11}$

Unido a estos serios pronósticos el Banco Interamericano de Desarrollo reporta que las remesas de los emigrados latinoamericanos declinarán el $11 \%$, lo cual es otro elemento negativo en este contexto. ${ }^{12}$ 
Y más recientemente en Octubre, en la reunión anual entre el Banco Mundial y el FMI, celebrada en Estambul, los pronósticos fueron más optimistas por un supuesto comienzo de la recuperación financiera, pero el economista jefe del FMI Olivier Blanchard dice que la crisis no ha terminado y que sus efectos negativos continuaran. ${ }^{13}$

Esta no es la primera vez que enfrentamos crisis, por lo que las experiencias vividas anteriormente han hecho que junto a las situaciones actuales y las perspectivas futuras hagan que la comunidad mundial se interese, con preocupación especial en el alcance y los efectos de la situación actual, dada su gravedad y globalidad.

Las aproximaciones ocurren desde las perspectivas políticas, económicas y sociales y desde los ámbitos específicos donde ocurren las mayores afectaciones: mercado laboral, salarios, alimentación, salud, industrias, agricultura, comercio, combustibles y otros. ${ }^{14,15}$

En el tema específico de la salud pública, existen temores de que la situación sanitaria se agrave a medida que el desempleo aumente, las redes de protección social empiecen a fallar, los ahorros y fondos de pensiones se vean erosionados, y el gasto e inversiones en salud disminuyan. También que aumenten las enfermedades mentales y la ansiedad, y que quizá se dispare el consumo de tabaco, alcohol y otras sustancias nocivas, como ha ocurrido en el pasado. ${ }^{3,4}$

En medio de este riesgoso escenario se encuentran los determinantes de la salud, todos altamente amenazados y muchos ya afectados, los servicios de salud con recortes financieros y el incremento de la demanda de atención, ya que las personas que se atendían en el sistema privado se dirigen al público, con la intención de ahorrar sus ingresos, en especial de problemas complejos y de alto costo, todo esto en sistemas fragmentados y/o segmentados que ya están sobresaturados y subfinanciados. ${ }^{16,17}$

Las amenazas para la salud son dramáticas para los países más pobres y vulnerables del mundo y sobre todo en las áreas que constituyen brechas dentro de los propios países donde se encuentran los bolsones de poblaciones más desfavorecidas y desprotegidas. Estas diferencias entre los países y dentro de ellos son mayores que en cualquier otro momento y un ejemplo es precisamente el continente americano, el más inequitativo de todos. ${ }^{18}$

\section{Los efectos de la crisis financiera sobre los Sistemas de Salud y las alertas y posibles soluciones ante la crisis}

Los efectos de la crisis financiera global sobre la salud, se pueden prever por las experiencias de crisis económicas anteriores, en las que a nivel mundial los gobiernos realizaron recortes de presupuestos y se afectó sobre manera el sector y donde se demostró un descuido en la atención a la salud y en particular en las acciones preventivas.

Los grupos sociales más vulnerables, mujeres, niños y adultos mayores, son los más afectados, tanto en su alimentación como en los cuidados generales y en servicios de salud, como consecuencia del incremento del desempleo y el aumento en el empleo informal o en el familiar no remunerado. Esto a su vez aumenta los niveles de pobreza, de desnutrición y la exclusión del acceso a los servicios básicos, lo que trae aparejado un incremento en los niveles de inequidad que ya por si solo es el principal problema histórico de la región de Las Américas, con la consiguiente 
afectación sobre la Ayuda Oficial al Desarrollo y el riesgo del no cumplimiento de los Objetivos de Desarrollo del Milenio. ${ }^{18-20}$

Varios organismos e instituciones del mundo se han proyectado con criterios prácticamente unánimes de que se debe respetar la inversión en el sector salud. La Dra. Margaret Chan, Directora de la Organización Mundial de la Salud, declaró:

El acceso a la atención de salud es un derecho fundamental y una responsabilidad de los gobiernos del mundo entero. Disminuir la exclusión, ampliar las prestaciones universales y proteger a la población frente al empobrecimiento, son elementos comunes de un número cada vez mayor de políticas nacionales de salud. Manejar las expectativas y contener la espiral de los costos de la atención de salud son aspectos decisivos, así como mantener la integridad de los sistemas que nos protegen de las amenazas transfronterizas, que detectan los brotes epidémicos, las pandemias y las enfermedades emergentes. ${ }^{21}$

En la II Reunión de Ministros de Salud del Movimiento de Países no Alineados se aprobó una declaración sobre crisis económica-financiera y salud, en la misma se expresó la preocupación ante la actual crisis y la perspectiva de que la asistencia oficial al desarrollo se redujera y que muchos países de bajos ingresos vean disminuida la ayuda internacional que reciben para múltiples programas sociales, en particular los que ejecutan en materia de salud.

La Dra. Chan ha pedido a los países ricos que no reduzcan la Ayuda Oficial al Desarrollo o que no recorten los gastos para la salud, la educación y la protección social. ${ }^{22}$

Cada vez, con mayor fuerza elementos como la solidaridad y la cooperación mundial salen más a la luz como soluciones colectivas a este fenómeno. Se acordó además la necesidad de reforzar las políticas públicas encaminadas a fomentar el pleno acceso a la salud y la protección social para los sectores más vulnerables de la sociedad y reconocieron la importancia de asumir las recomendaciones realizadas por la Comisión sobre Determinantes Sociales de la Salud, que se enfocan en tres principios de acción:

1. Mejorar las condiciones de vida y de trabajo y formular políticas de protección social dirigidas a toda la población y crear las condiciones que le permitan envejecer bien. Estas políticas deben contar con la participación de la sociedad civil, los poderes públicos y las instituciones internacionales.

2. Luchar contra la distribución desigual del poder, el dinero y los recursos, para combatir la inequidad sanitaria y las disparidades en las condiciones de vida.

3. Medir la magnitud del problema, analizarlo y evaluar el efecto de las intervenciones. Reconocer que existe un problema y conseguir que se evalúe la magnitud de la inequidad sanitaria a nivel nacional y mundial es un punto de partida esencial para la acción. Ampliar la base de conocimientos y dotarse de personal capacitado en materia de determinantes sociales de la salud y sensibilizar a la opinión pública a ese respecto, es otro aspecto fundamental. ${ }^{23}$

La Comisión explica que el enfoque en los determinantes sociales de salud, es necesario porque las condiciones sociales en que viven las poblaciones tienen un gran impacto en la salud. De hecho, circunstancias como la pobreza, la discriminación social, malas condiciones de vivienda, deficiente niveles de salud en la niñez y pocas posibilidades de empleo en la adultez, son los mayores factores de inequidad entre y hacia el interior de los países en términos de salud, enfermedades y tasas de mortalidad. ${ }^{24-26}$ 
En el marco de la II Reunión de Ministros de Salud del Movimiento de Países no Alineados los países miembros se trazaron objetivos de trabajo encaminados a:

- La necesidad de preservar e incrementar los presupuestos dedicados a la salud.

. Fortalecer los sistemas nacionales de salud.

. Fortalecer los enfoques de atención primaria de salud.

- Tomar medidas para garantizar el acceso de toda la población a los servicios de salud y medicamentos.

- Dirigir la cooperación internacional hacia el desarrollo y mantenimiento de los sistemas de salud, transferencia de tecnologías, fomentar capacidades nacionales y hacia el logro de los Objetivos de Desarrollo del Milenio. ${ }^{27}$

La posición planteada en la Región por la Organización Panamericana de la Salud, es la de impulsar la estrategia de Atención Primaria de Salud (APS) como base de los sistemas, se considera que la renovación de la APS debe ser parte integral de su desarrollo y que a su vez los sistemas de salud basados en esa estrategia son los mejores para promover mejoras equitativas y sostenible de la salud de los pueblos. La OPS orienta que hacer para ir hacia la calidad, responsabilidad, justicia social, sostenibilidad, participación e intersectorialidad. ${ }^{28}$

Se identifican cuatro áreas para el trabajo en la atención primaria de salud renovada: cobertura universal de los sistemas de salud para alcanzar la equidad en salud, liderazgo para hacer a las autoridades de salud más confiables, lograr que el centro de los sistemas sea la población y establecer políticas públicas que promuevan y protejan la salud de las comunidades. ${ }^{29}$

Además se realiza un llamado por parte de la Organización Panamericana de la Salud, hacia la creación y fortalecimiento de las alianzas que permitan maximizar el resultados de los esfuerzos individuales, ya sean bilaterales, subregionales, ONGs o sector privado, con la participación comunitaria como elemento fundamental.

Otro elemento es el desarrollo de redes de seguridad social que protejan a las poblaciones vulnerables de los países ricos y pobres, con la distribución equitativa de la atención de salud y cobertura universal que no solo protege a los pobres, sino que es el modo más asequible y eficiente de utilizar los recursos cuando escasean. ${ }^{19}$

Cuando se aplica adecuadamente la estrategia de APS, ofrece protección frente a muchos de esos problemas, influye en los determinantes de la salud fundamentales que proceden de múltiples sectores externos al de la salud, ataca precozmente a las amenazas, devuelve el equilibrio a la atención sanitaria, y sitúa a las familias y las comunidades en el centro del sistema de salud. Hace hincapié en la participación social, el empoderamiento de la comunidad que al hacer suyos los problemas de salud participa en su solución.

Se ha demostrado que la vía para afrontar las inequidades es avanzar hacia la cobertura universal con un espíritu de equidad, justicia social y solidaridad. La justicia y la eficiencia en la prestación de los servicios constituyen los objetivos generales.

La APS ofrece asimismo la mejor posibilidad de afrontar tres males de la vida del siglo XXI: la globalización de los modos de vida insalubres, la aceleración de la urbanización no planificada y el envejecimiento de las poblaciones. ${ }^{30,31} \mathrm{Su}$ planteamiento multisectorial es un elemento capital de la prevención y promoción de la salud, habida cuenta de que los principales factores de riesgo de esas enfermedades son externos al sector de la salud. 
Esos aspectos han sido ampliamente difundidos y defendidos en la arena internacional al celebrarse el 30 Aniversario de la Declaración del Alma Atá y el Informe Mundial 2008 de la OMS está dedicado a la importancia de la APS. ${ }^{32}$

Otro importante esfuerzo por demostrar que la APS es una vía para proteger a las poblaciones de los efectos de la crisis sobre su salud, es el proyecto de Resolución sobre Atención Primaria de Salud y el fortalecimiento de los sistemas de salud, presentado en la 62 Asamblea Mundial de la Salud, donde se propone que se elabore un plan de acción con miras a presentarlo en la 63 Asamblea Mundial en el año 2010 y en el que se recojan las estrategias de los países para establecer las reformas en los sistemas de salud que permitan la renovación de la APS. ${ }^{33}$

\section{Experiencias de Cuba de crisis anteriores}

Cuba, con un modelo de desarrollo de carácter socialista, donde la salud es un derecho de todos los ciudadanos y es una responsabilidad y prioridad del Estado como se recoge en la constitución de la República en el artículo 50 y en la Ley 41 de Salud Pública, posee un sólido Sistema Nacional de Salud, basado en los principios de ser: universal, gratuito, accesible, regionalizado, integral y con una concepción internacionalista, presenta una férrea voluntad política de perfeccionarlo cada día más. Muestra indicadores que así lo demuestran: mortalidad infantil 4,7 x 1000 nacidos vivos, mortalidad en menores de 5 años de 6,2, esperanza de vida al nacer de 78 años, 13 enfermedades protegidas por vacunas, la mayoría producidas en Cuba, y un grupo de enfermedades eliminadas. ${ }^{34}$

En el año 1989 después de la caída del campo socialista de Europa del Este, el país sufrió un serio periodo de crisis al cual se le denominó "Periodo Especial", se produjo una caída abrupta de su capacidad importadora en el $75 \%$ y la exportadora en el $73 \%$. El PIB descendió a casi el $35 \%$ con un déficit presupuestario de $33 \%$ que generó fuertes contracciones en los programas económicos y sociales. La tasa de inversiones descendió del 26 al 7\%. Además se recrudeció el bloqueo de Estados Unidos, vigente desde 1962.

Las repercusiones fueron visibles en todas las esferas de la sociedad: dentro de las sociales se enmarcó la reducción de las capacidades de empleo, se afectó la alimentación por la carencia de productos y la inestabilidad de las ofertas y altos precios.

El ambiente también fue dañado en cuanto a la disponibilidad y calidad del agua potable, la recogida y disposición de los desechos sólidos, el saneamiento básico, se incrementaron los vectores y la falta de higiene.

El combustible se redujo a límites extremadamente bajos, las importaciones de petróleo cayeron a menos de la mitad, se afectó notablemente el transporte, así como la generación de electricidad y la preparación de alimentos.

El sector educacional sufrió un descenso de casi el $20 \%$, con la consiguiente carencia de libros, útiles escolares, deterioro de las estructuras de los inmuebles, entre otros.

El Sistema Nacional de Salud no estuvo ajeno a estas afectaciones, sufrió recortes en recursos financieros y materiales, afectaciones en el clima laboral, en la organización y gestión de los servicios, en la situación de salud de la población, en su satisfacción con las prestaciones y la reducción en el 70 \% del presupuesto en divisas convertibles, sin embargo, se mantuvo el incremento anual del presupuesto en moneda nacional y el gasto por habitante reorientado en APS. ${ }^{35}$ 
El gasto en salud como porcentaje del PIB y del presupuesto estatal siempre mantuvo una tendencia creciente, esta estrategia fue la expresión de la prioridad concedida al sector de la salud.

En relación con la salud de la población, las afectaciones no se correspondieron con la gravedad económica y social de la crisis, mientras el PIB decrecía en el $35 \%$ y el presupuesto disminuía cerca del $70 \%$, la mortalidad infantil descendió en casi el 15 $\%$, la mortalidad del menor del 5 años en más del $10 \%$ y la mortalidad materna en el $8 \%$. Además, se logró mantener los principales servicios de salud, principalmente en la atención primaria de salud.

Hubo un impacto negativo en los servicios, en la atención secundaria y otros como la disminución de los ingresos hospitalarios, las operaciones quirúrgicas electivas, las consultas estomatológicas, las donaciones de sangre, los exámenes diagnósticos, disponibilidad de medicamentos, reactivos, productos y suministros médicos; el transporte por ambulancias y el mantenimiento de las infraestructuras, mobiliario y equipamiento de las unidades del sistema, en especial los hospitales.

Sin embargo, se incrementaron las capacidades y utilización de los hogares maternos como servicios de protección a la embarazada y al recién nacido en los aspectos nutricionales, higiénicos y de atención médica.

En estas condiciones el país se vio obligado a aplicar un fuerte proceso de ajustes, que constituyó la respuesta social y estatal a la crisis económica, con el propósito de evitar que se lesionaran las bases y los logros, políticos y sociales alcanzados sin tener que recurrir a fórmulas neoliberales aplicadas en otros países afectados por crisis similares. ${ }^{35,36}$

Las estrategias adoptadas en salud, se caracterizaron por combinar, no sin contradicciones, la centralización de las decisiones estratégicas y la descentralización de las decisiones tácticas y operativas, con participación estrecha de las estructuras del gobierno, el partido y la comunidad, para situarlas dentro del contexto político, económico y social del país.

Esta etapa difícil obligó a tomar medidas excepcionales de subsistencia y sobrevivencia para poder salvar al país y donde se afectaron muchos sectores de la población, pero sin embargo, en el caso de la salud a pesar de las lógicas e inevitables consecuencias se pudo, en lo fundamental preservar lo avanzado en años anteriores y significó una gran experiencia, válida para cualquier tipo de crisis.

A partir del año 1994 se inicia la recuperación económica, con un conjunto de medidas adoptadas, se reanimaron varios sectores de la economía como: el turismo, la agricultura y las construcciones, así como la generación de empleos directos e indirectos. Se desarrolló un nuevo sector de alta tecnología entre ellos productos biotecnológicos, farmacéuticos, equipos médicos, servicios médicos de alta complejidad y los productos y servicios informáticos de alto valor añadido, exportación de servicios, donde el sector salud tiene un peso relevante.

En lo social, las medidas adoptadas permitieron mantener a los trabajadores en sus empleos y conservarles sus ingresos nominales a ellos y a los pensionados por el sistema de seguridad y asistencia social junto a la preservación de los programas de salud y educación o sea, proteger de la exclusión a los distintos grupos de la población. ${ }^{35,36}$

En un segundo momento las medidas fueron en busca de la eficiencia y aquí existen dos etapas: 
Desde 1993-2000, transformaciones en el diseño de los sistemas de prestación de servicios, descentralización, modernización y fortalecimiento de las instancias locales y nuevos matices respecto a la participación de instituciones sin fines de lucro en la gestión y financiamiento de la política social, ${ }^{37}$ y a partir del año 2001 , se inicia un conjunto de programas especiales que se enfocan a incrementar la calidad de la atención y su descentralización, acercando los servicios a la comunidad. Estos programas intentan alcanzar un nivel de excelencia en las prestaciones mediante un desarrollo superior de la promoción de salud, la prevención de las enfermedades, así como las acciones de recuperación y rehabilitación. Dentro de los principales programas desarrollados se encuentran:

1. Programa Nacional de Salud y Calidad de Vida, dirigido a lograr hábitos dentro de la población para reducir los principales factores de riesgo y lograr estilos de vida más saludables.

2. Policlínico integral. Se reduce el número de casos que llegan a los hospitales y se descentralizan un conjunto de servicios de rehabilitación y fisioterapia que garantizan la atención de un grupo de pacientes en sus propias comunidades.

3. Hospitales de excelencia, con equipos de alta tecnología.

4. Programa de formación de enfermeras.

5. Formación de tecnólogos de la salud.

6. Reparación de farmacias y su nueva función, más asistencial y menos comercial, con una mayor calidad en el servicio.

7. Importación de equipos de alta tecnología para perfeccionar los servicios oftalmológicos del país.

8. Rehabilitación de 118 salas de terapia intensiva en policlínicos en comunidades donde no existen hospitales, 24 nuevos servicios de hemodiálisis y 88 ópticas.

9. Cooperación Internacional en diferentes latitudes del planeta, Programa Integral de salud y Operación Milagro.

10. Formación de médicos en la Escuela Latinoamericana de Medicina.

11. Creación del destacamento internacional "Henrry Reeve" para prestar asistencia en grandes desastres y epidemias. ${ }^{38,39}$

Sin duda, todas las acciones tomadas al final de la década de los 90, basadas fundamentalmente en: reafirmar la APS y sus componentes como el modelo esencial para el sistema de salud, la participación comunitaria y la intersectorialidad, como estrategia participativa y el fortalecimiento del papel rector de las estructuras y niveles del sistema con autoridad descentralizada, fueron entre otras, las que permitieron la sostenibilidad y preservación de los logros alcanzados y han servido también como lecciones aprendidas para fortalecer la recuperación del país en período de crisis. ${ }^{35,39}$

\section{Preparación del Sistema Nacional de Salud ante la crisis}

El país no está ajeno a la situación mundial, actualmente se encuentra en un momento difícil desde el punto de vista económico, sufrió el impacto catastrófico de tres huracanes, con un costo aproximado de 10 mil millones de USD, equivalente al $20 \%$ del PIB y se mantienen las condiciones de bloqueo económico que ha costado más de 96 mil millones de dólares hasta el 2008, por lo que se requiere elevar la capacidad de previsión y análisis en todos los niveles del sector.

El impacto de la actual crisis ya está presente, por ejemplo, de un crecimiento económico estimado inicialmente para el año 2009 de $6 \%$, ahora se estima disminuya hasta el 1,7\%. Las causas fundamentales son la disminución de las exportaciones y sobre todo los bajos precios del níquel, principal renglón exportable; la disminución en el $13 \%$ de los ingresos por la vía del el turismo, a pesar del incremento en el 3,9\% en el número de turistas.Además, los altos 
precios de las importaciones de alimentos, combustibles y otros, o sea, una disminución ostensible en el intercambio comercial que incluye una disminución en las remesas recibidas. ${ }^{40}$

En estudios realizados se han identificado tres momentos importantes ante una crisis económica y sus efectos sobre la salud: el período íntercrisis, o el antes, el momento del enfrentamiento, o el durante, y la etapa de recuperación, o el después. Todas de igual importancia aunque con sus peculiaridades y formas de atención. ${ }^{35}$

En este sentido y como elemento de fortalecimiento del sistema, desde el año 2006 se asumió un nuevo período programático de diez años, el mismo definió las proyecciones, prioridades, acciones y metas del Ministerio de Salud Pública, se denominó Proyecciones de la Salud Pública cubana hasta el 2015, las mismas se orientan a los objetivos siguientes:

1. Lograr una adecuada intersectorialidad, acciones que influyan de forma favorable en el ambiente y con ello una protección superior a la salud de la población.

2. Desarrollar con efectividad un trabajo dirigido a la disminución y control de los factores de riesgo más importantes que afectan a la salud de la población.

3. Disminuir la mortalidad y morbilidad de las enfermedades no transmisibles y otros daños a la salud que constituyan las principales causas de enfermedad y muerte en la población cubana.

4. Mantener y mejorar la situación de salud alcanzada en las enfermedades infecciosas y parasitarias y hacer énfasis en aquellas que no se han podido resolver mediante la aplicación de inmunobiológicos y en las exóticas susceptibles de introducirse en el país.

5. Consolidar y mejorar los niveles alcanzados en la salud de la madre y el niño.

6. Desarrollar las acciones que permitan enfrentar con éxito la atención que demanda el envejecimiento de la población cubana. ${ }^{38}$

Ante esta situación, el Ministerio de Salud Pública ha enfocado su política de mantener y consolidar la organización y estabilidad de los servicios a la población, garantizar la protección ante la amenaza de epidemias, la calidad de la formación de recursos humanos y de las actividades que generan ingresos de divisas al país (exportación de servicios).

Se han elaborado una serie de medidas dentro de las que se encuentran:

- Trazar políticas que aseguren planes alcanzables, a partir del respaldo financiero, material y de fuerzas de trabajo.

- Reducir gastos adecuados a los ingresos.

. Posponer inversiones y otros gastos no imprescindibles.

. Incrementar producciones alternativas, principalmente alimentos.

. Establecer reservas que permitan enfrentar situaciones imprevistas. ${ }^{41}$

Las experiencias de crisis anteriores permiten aseverar que la inversión social, en etapas de intercrisis, constituye la intervención de elección para mejorar la salud y sus determinantes y para enfrentar y mitigar los efectos sobre la salud que ellas producen. De igual forma las políticas basadas en el principio de justicia social, expresadas en la igualdad de derechos al acceso a bienes y servicios, con equidad en su distribución, que privilegie a los grupos más vulnerables, para obtener igualdad de resultados en toda la población, constituyen la condición esencial para la protección de la población, antes, durante y en la recuperación de la crisis. ${ }^{35}$

\section{La Atención Primaria de Salud y formación de recursos humanos}

http://scielo.sld.cu 
Cuba, es un ejemplo en el desarrollo y aplicación de la estrategia de la APS,como base del Sistema Nacional de Salud. Desde 1975 ya se tenía una concepción de cuales debían ser las características esenciales de un modelo de atención médica en la comunidad, que aún hoy en día es:

. Integral: unidad de los aspectos preventivos y curativos.

- Sectorizado: responsabiliza al médico con un número determinado de habitantes.

- Regionalizado: establece la interrelación de los distintos niveles de organización.

. Continuo: atención a lo largo del tiempo y en todas las actividades del área de salud.

. Dispensarizado: agrupación, registro y control periódico de afecciones y riesgos.

. En equipo: acciones conjuntas interdisciplinarias.

. Con participación activa de la comunidad.

Esta estrategia se perfecciona y desarrolla con el transcurso del tiempo, se incorporan avances tecnológicos y se mejora la calidad del servicio que se le brinda a la población, acercándolos y evitando saturar la atención secundaria. Hoy en el Sistema Nacional de Salud se brindan los siguientes servicios en la atención primaria de salud.

. Rehabilitación Integral.

- Electrocardiografía de urgencia y electiva.

- Radiología.

. Ultrasonido.

- Optometría.

. Oftalmología.

. Endoscopia.

. Apoyo vital y trombolisis.

- Traumatología.

- Laboratorio clínico.

- Laboratorio de alergia.

- Drenaje biliar.

. Regulación menstrual.

. Planificación familiar.

. Esterilización.

. Estomatología.

- Atención integral a la mujer y al niño.

. Inmunizaciones.

- Atención integral al paciente diabético.

- Atención integral al paciente con cáncer.

. Atención integral al adulto mayor.

- Cirugía menor y mayor.

. Quimioterapia.

. Áreas intensivas municipales.

. Audiometría.

. Salón de partos.

. Sala para ingreso.

. Donación de sangre.

. Genética clínica. ${ }^{39}$

Además se convirtió en el escenario principal de formación de los recursos humanos para el sector salud, donde se estudian las carreras de medicina, estomatología, psicología, enfermería y tecnologías de la salud.

Surge a nivel local la concepción del Policlínico Universitario como parte de las transformaciones que tienen lugar en la enseñanza de la medicina en Cuba. La 
validez de este nuevo modelo de formación de médicos donde a nivel del policlínico se funden la asistencia, la docencia y la investigación, ratifica a la APS como soporte esencial del sistema.

Las sedes universitarias constituyen una estructura organizativa ubicada en los municipios donde los estudiantes residen, estudian y laboran. Es un escenario de trabajo convertido en Universidad, este proceso transformador produce cambios en el orden cualitativo y demuestra la base técnica y científica desarrollada, así como la capacidad docente de que dispone el país. ${ }^{42}$

Todo lo anterior se ha desarrollado, entre otras cosas, porque en el país existe un total de 566365 trabajadores de la salud, de ellos médicos 74552 y especialistas en Medicina General Integral 32 289, con una proporción de 1 médico por 151 habitantes y una amplia red de instituciones de salud: consultorios del médico de familia para el $100 \%$ de la población, policlínicos, hospitales, institutos especializados y otros. ${ }^{42,43}$

Este escenario permite la formación no solo de profesionales cubanos sino también constituye la base formativa para muchos estudiantes extranjeros, que de forma solidaria se preparan en Cuba. Con esta experiencia se transforma el paradigma de la enseñanza médica en el mundo, los médicos regresan a sus países con un enfoque distinto del ser humano y de la medicina cubana. Se pretende graduar para el 2013 aproximadamente 61632 médicos extranjeros y esto sin duda contribuirá a fortalecer las capacidades nacionales de países hermanos y contribuir al logro de objetivos comunes de salud para el mundo. ${ }^{42}$

\section{Escenario hipotético sobre el impacto de la crisis financiera mundial en el sistema de salud cubano, sin el bloqueo económico, financiero y comercial de Estados Unidos sobre Cuba}

El bloqueo de Estados Unidos contra Cuba tiene una historia de casi 50 años, según memorando clasificado comenzó el 6 de abril de 1960.

Han sido medidas de presión política contra el gobierno de Cuba, pero solo para dañar a la población, la cual ha sufrido desde entonces, pero al mismo tiempo ha resistido a pesar de sus nefastas consecuencias.

A lo largo del tiempo, los mecanismos restrictivos del bloqueo se incrementaron, surgieron nuevas leyes: la Ley para la Democracia Cubana conocida por Ley Torricelli en 1992 y la Ley por la Solidaridad Democrática y Libertad Cubana, conocida como Ley Helms-Burton en 1996, que nada tienen que ver con sus eufemísticos nombres, pues sus reales objetivos son reforzar las medidas económicas contra Cuba y sustentar el carácter extraterritorial del bloqueo, ya que prohibieron a compañías subsidiaras norteamericanas en terceros países realizar transacciones con Cuba o nacionales cubanos, o denegaron la entrada a Estados Unidos a directivos y familiares de empresas extranjeras que inviertan en Cuba e incluso presentar demandas en su contra en los tribunales de EE.UU, entre otras medidas. ${ }^{44}$

Desde el año 1992, la Asamblea General de Naciones Unidas ha debatido este tema a propuesta de Cuba en 18 ocasiones y siempre han condenado el bloqueo e incluso el 28 de octubre de 2009, votaron a favor de eliminar el bloqueo 187 países y solo 3 en contra, que fueron Estados Unidos, Israel y Palau. ${ }^{45}$

Esto demuestra que la comunidad internacional está en contra del bloqueo y de la aplicación de estas medidas económicas coercitivas y leyes extraterritoriales y que 
el resultado de este ejercicio anual de política exterior es un fracaso total para Estados Unidos, al quedar prácticamente aislado y solo en la aplicación de estas absurdas e irracionales medidas contra Cuba.

Por otro lado, según resultados de encuestas recientes de instituciones propias de ese país, indican que el $76 \%$ de los norteamericanos se oponen al bloqueo. ${ }^{46}$

Entonces, ¿por qué continúa el bloqueo?

Las afectaciones al Sistema de Salud cubano y sus instituciones, son considerables y es lógico si se tiene en cuenta los propósitos declarados del bloqueo de producir hambre y enfermedades como supuesto medio de presión política.

Se sabe que durante estos años las pérdidas calculadas de la economía cubana alcanzan los 96 mil millones de dólares, unos 236 mil 221 millones, de acuerdo a los precios actuales del dólar norteamericano y en el caso de salud son más de 2 mil 400 millones. Aunque estos valores por si solo no expresan el nivel de sufrimiento y angustia ocasionado, si permiten imaginar el progreso que Cuba habría alcanzado sin la existencia del bloqueo que obliga a comprar equipos y productos imprescindibles para la salud de la población en mercados lejanos, con utilización de intermediarios, con incremento de los costos y del tiempo, algo muy grave cuando se habla de salvar vidas y dar salud. ${ }^{47}$

Otro aspecto muy negativo, es el no otorgamiento de visas a científicos y especialistas cubanos de la salud para participar en numerosos eventos y congresos científicos en Estados Unidos.

Hay ejemplos demostrados de la negación de compras de medicamentos y productos con carácter urgente para luchar contra epidemias, como fue en 1981, cuando se enfrentó una misteriosa epidemia de dengue hemorrágico con más de 300 mil casos.

Actualmente hay otros ejemplos específicos como son:

- Obligan a la realización de operaciones de niños a corazón abierto, porque se prohíbe por Estados Unidos, vender a Cuba dispositivos para el cateterismo pediátrico.

. Prohibición a la Compañía Merck de vender medicamentos como el "Elspar" para niños que padecen de leucemia linfoblástica.

. No autorización para adquirir un equipo de Analizador de Genes, para el estudio del origen del cáncer de mama, de colon y de próstata.

- Incumplimiento de la Philips Medical de suministro de piezas de repuesto para equipos médicos comprados por 72 millones de dólares, por presiones y multas del gobierno de Estados Unidos, a pesar de ser una compañía de Países Bajos, lo cual es un ejemplo de la extraterritorialidad del bloqueo.

- Las compañías Hitachi y Toshiba declararon que no pueden vender a Cuba un microscopio de trasmisión electrónica y equipos como Cámara Gamma, de Resonancia Magnética, Ultrasonidos de alta precisión y otros, por presiones de Estados Unidos a pesar de ser japonesas. ${ }^{47}$

En la actualidad el Sistema de Salud cubano enfrenta las consecuencias de la crisis económica global y también un feroz bloqueo, rechazado y condenado por la comunidad internacional, dirigido fundamentalmente a dañar la salud de la población cubana. 
A pesar de ello, por contar con una amplia red de instituciones de servicios médicos, con recursos humanos suficientes y capacitados y de un Sistema de Salud con base en la atención primaria de salud, como ya se ha expuesto, se mantienen excelentes indicadores de salud. Es lo que algunos autores han Ilamado "la paradoja de la salud cubana. ${ }^{48}$

Cuestión reconocida y destacada también por la directora general de la OMS en una intervención especial durante su reciente visita al Instituto de Medicina Tropical "Pedro Kourí", cuando dijo:

...la colaboración internacional ante una emergencia de salud, no debe ser afectada por ninguna barrera política. Soy consiente del impacto económico, comercial y financiero que el embargo tiene sobre este país. Bajo estas circunstancias, lo que ustedes han alcanzado en términos de acceso universal a la salud, resultados sobresalientes de la salud y la ayuda médica a otros países de América Latina y África es realmente sorprendente. ${ }^{49}$

Pero lo anterior, no seria posible si no fuera por la prioridad que da el gobierno a la salud y la voluntad política para defender y mantener las conquistas alcanzadas, ante cualquier tipo de crisis.

Por tanto, no es difícil imaginar un escenario de la salud cubana sin el bloqueo, donde el sistema tuviera garantizado lo que necesita, de forma ágil, con la calidad requerida y con menos costos, sin obstáculos que obligan a múltiples y complejas gestiones y esfuerzos adicionales al país. Seguro se hubiese evitado mucho sufrimiento, carencias y daños, que hoy en día continúan afectando a todos.

\section{LECCIONES APRENDIDAS}

El mundo ha tenido diferentes momentos de crisis y cada país en cuestión ha diseñado sus estrategias para salir o recuperarse de ella. Existen aspectos básicos, que resultan obligados mencionar una vez que se esboza la forma en que Cuba enfrentó la crisis en materia de salud, dentro de estas se encuentran:

. La salud de la población debe estar en manos del Estado, ser universal y gratuita para todos los ciudadanos.

- La voluntad política del gobierno es quien determina en última instancia que no se recorten los recursos destinados a salud.

- La integridad e intersectorialidad entre las instituciones de salud, el resto de los organismos involucrados y las diferentes organizaciones de la sociedad cubana, son factores clave para desarrollar acciones que optimizan los pocos recursos y potencian los resultados a favor de su eficiencia y efectividad.

. Los Sistema de Salud basado en la estrategia de APS permiten potenciar el trabajo preventivo en las comunidades, descentralizar los servicios médicos acercándolos a las comunidades e implementar programas personalizados. - La decisión de adelantar el desarrollo social al económico en algunas etapas del desarrollo cubano, implica un alto grado de justicia social y ciudadana.

. Formación continua y especializada de médicos, tecnólogos y paramédicos.

. Desarrollo de la industria farmacéutica, biotecnológica, vacunas, equipos y de software dirigido a la salud, fundamentalmente a resolver los principales problemas de la población.

- La oportunidad que representa la crisis para mejorar la eficacia y eficiencia del trabajo.

Es un hecho que la actual crisis global, impacta la economía cubana, por demás subdesarrollada y bloqueada, pero la organización, estrategia y acciones 
desarrolladas por el sistema de salud cubano, pone al sector en mejores condiciones para enfrentar los retos que de la crisis se deriven, de forma tal que se continúe dando respuestas a las demandas de la población. Esto implica que Cuba está preparada para enfrentar la crisis financiera y económica actual, la cual todos los países en alguna medida tendrán que enfrentar.

\section{CONSIDERACIONES FINALES}

Las actuales crisis que vive el mundo repercuten en la salud, pero más aún en la económica financiera. La principal preocupación a nivel internacional radica en la disminución en los gastos en salud y con ello el incremento de las desigualdades sociales y la inequidad que ya son una triste realidad. La estrategia de Atención Primaria de Salud demuestra que es una solución viable por los países para superar estas diferencias, dar cobertura universal y alcanzar los Objetivos de Desarrollo del Milenio, no solo por la aceptación de los Organismos Internacionales a esta estrategia, sino con el propio ejemplo del sistema de salud cubano durante 50 años, que ostenta indicadores de salud comparables con países del primer mundo.

La experiencia cubana de crisis anteriores demuestra que un país requiere de inversión social, para mejorar la salud y sus determinantes y para enfrentar y mitigar los efectos sobre la salud que ellos producen. Las políticas basadas en el principio de justicia social, expresadas en la igualdad de derechos al acceso a bienes y servicios con equidad en su distribución, que privilegie a los grupos más vulnerables para obtener igualdad de resultados en toda la población, constituyen la condición esencial para la protección de la población, antes, durante y en la recuperación de la crisis. La formación de recursos humanos sobre los principios y valores éticos del derecho a la salud, permiten preparar el capital humano necesario para alcanzar un verdadero desarrollo en la salud de las poblaciones.

En estos momentos se requiere de solidaridad internacional, de compartir experiencias y de unidad entre los pueblos para enfrentar no solo la crisis financiera que afecta a todos los países, sino las demás que refuerzan sus efectos negativos, como son: la crisis alimentaria, de recursos humanos, energética y el cambio climático.

El bloqueo económico, comercial y financiero que el gobierno de Estados Unidos ejerce sobre Cuba, refuerza sobre el país los efectos negativos de la crisis económica global, este es un ejemplo de la repercusión a nivel económico y social de la política exterior de un país sobre una nación y de la inmunidad de esta acción, aunque reciba un rechazo universal.

\section{REFERENCIAS BIBLIOGRÁFICAS}

1. Ravishankar N. Financing of global health: tracking development assistance for health from 1990 to 2007. Lancet. 2009;373:2113-24.

2. Chan M. Discurso pronunciado en la 62 Asamblea Mundial de la Salud. 19 de mayo de 2009. Ginebra, Suiza [sitio en Internet]. [citado 16 Oct 2009]. Disponible en: http://apps.who.int/gb/ebwha/pdf files/WHA62-REC1/WHA62 REC1-sp-P1.pdf 
3. Discurso pronunciado en la II Reunión de Ministros de Salud del MNOAL. 20 de mayo de 2009. Ginebra: OMS; 2009.

4. . El efecto de las crisis mundiales en la salud: dinero, clima y microbios. Tercer foro sobre asuntos de interés mundial. 18 de marzo de 2009. Berlín, Alemania [sitio en Internet]. [citado 16 Oct 2009]. Disponible en: http://www.who.int/dg/speeches/2009/finalcial crisis 20090318/es/index.html

5. Chomsky. El Neoliberalismo, raíz común de las crisis actuales. Discurso pronunciado en la Iglesia Riverside, 12 Junio 2009. La Jornada, en digital [sitio en Internet]. [citado 15 Jun 2009]. Disponible en: http://www.jornada.unam.mx/ultimas/

6. Martínez O. Crisis económica global. ¿Hasta cuándo? ¿Hasta dónde? Granma digital [sitio en Internet]. [citado $27 \mathrm{Abr}$ 2009]. Disponible en:

http://www.granma.cubaweb.cu/2009/04/27/interna/artic01.html

7. Riera L. Desempleo crece sin tregua en los E.U. Granma digital [sitio en Internet]. [citado 7 Abr 2009]. Disponible en: http://www.granma.cubaweb.cu/

8. González L. Retrocederá más la economía mundial, pronostica la ONU [sitio en Internet]. [citado 9 May 2009]. Disponible en: http://www.granma.cubaweb.cu/2009/05/28/interna/artic01.html

9. Justo M. Las crisis invisibles. Granma digital [sitio en Internet]. [citado $18 \mathrm{Sept}$ 2009]. Disponible en:

http://www.granma.cubaweb.cu/2009/09/18/interna/artic03.html

10. Marmot MG. Feature. How will the financial crisis affect health? BMJ. 2009;388:b1314.

11. Porto M. La seguridad alimentaria, ¿es posible? Granma digital [sitio en Internet]. [citado 23 Jun 2009]. Disponible en:

http://www.granma.cubaweb.cu/2010/02/19/interna/index.html

12. Banco Interamericano de Desarrollo. Las remesas en tiempo de inestabilidad financiera. El impacto de la crisis financiera sobre las remesas a América Latina y El Caribe [sitio en Internet]. [citado 18 Nov 2009]. Disponible en:

http://idbdocs.iadb.org/wsdocs/getdocument.aspx?docnum $=1913744$

13. FMI. La economía global se recupera pero no tan rápido como antes. Nuestro País, endigital [sitio en Internet]. [citado 3 Oct 2009]. Disponible en: http://www.elpais.co.cr/

14. World Bank. World Bank Praises Latin America's Resilience in Global Financial Crisis [sitio en Internet]. [citado 6 Jul 2009]. Disponible en: http://go.worldbank.org/3MXFWHG480

15. Kohn L. Global Economic Integration and Decoupling. Speech at the international research forum on Monetary Policy, Frankfurt, Germany. June 26, 2008 [sitio en Internet]. [citado 10 Nov 2009]. Disponible en: http://www.federalreserve.gov/newevents/speech/kohn20080626a.htm

16. Jack A. Feature Commentary: Look after de pennies. BMJ. 2009;338: b 1380. 
17. Commission on Social Determinants of Health. Final report-: closing the gap in a generation: health equity through action on the social determinants of health. Geneva: WHO [sitio en Internet]. 2008 [citado 10 Nov 2009]. Disponible en: http://www.who.int/social determinants/thecommission/finalreport/en/index.html

18. Akerman M. Social Determinats of health: Is this an international Issue? Washington, D.C.: OPS, 2009.

19. Fondo Fiduciario España-PNUD. Entrevista exclusiva a Mirtha Roses, Directora de la Organización Panamericana de la Salud [sitio en Internet]. 2008 [citado 16 Oct 2009]. Disponible en:

http://devserver.paho.org/equity/index2.php?option=com docman\&task=doc view \&gid $=16 \&$ Itemid $=159$

20. Organización Panamericana de la Salud. La recesión mundial y los Objetivos de Desarrollo del Milenio. ¿De qué manera la crisis económica puede afectar el cumplimiento de los ODM? Washington, D.C.: OPS;2009.

21. Chan M. Palabras pronunciadas en la región consultiva de alto nivel sobre la crisis financiera y la salud. Ginebra: OMS; 2009.

22. Reunión Consultiva de Alto Nivel sobre la Crisis Financiera y Económica y la Salud Mundial. La crisis financiera y la salud mundial. Ginebra, Suiza. 16 de enero de 2009 [sitio en Internet]. [citado 23 Oct 2009]. Disponible en: http://www.who.int/mediacentre/events/meetings/finalcial crisis 20090113/es/ind ex.html

23. Comisión sobre Determinantes Sociales de la salud. Resumen analítico del Informe Final. Subsanar las desigualdades en una generación. Alcanzar la equidad sanitaria actuando sobre las determinantes sociales de la salud. OMS. Ginebra, Suiza, 2008 [sitio en Internet]. [citado 16 Oct 2009]. Disponible en: http://www.who.int/social determinants/strategy/QandAs/es/index.html

24. Commission on Social Determinants of health. Action on the social determinants of health: Learning from previous experiences (March). Geneva: WHO; 2005.

25. Gottret P. Protecting Pro-Poor Health Services during Financial Crisis. Lessons from Experience. April 2009 [sitio en Internet]. [citado 10 Nov 2009]. Disponible en:

http://siteresources.worldbank.org/HEALTHNUTRITIONANDPOPULATION/Resources $\angle$ ProtectingProPoorFC.pdf

26. Health amid a financial crisis: a complex diagnosis. Bull WHO. 2009;87(1):4-5.

27. Buró Coordinador del Movimiento de Países no Alineados. Declaración sobre crisis económica financiera y la salud. II Reunión II Reunión Ministerial de Ministros de Salud del MNOAL. Ginebra, Suiza, 20 de mayo 2009[sitio en Internet]. [citado 16 Oct 2009]. Disponible en: http://72.249.12.201/wordpress$\mathrm{mu} / \mathrm{cuba} /$ files/2009/05/declaracion-final-traduccion-espanol.doc

28. Documento de Posición de la Organización Panamericana de la Salud/Organización Mundial de la Salud. La renovación de la Atención Primaria de Salud en las Américas. Washington, D.C: OPS;2007. 
29. Macinko J, Montenegro $\mathrm{H}$, Nebot $\mathrm{C}$. La renovación de la Atención Primaria de Salud en las Américas. Rev Panamericana Salud Pública. 2007; 21(2/3):73-84.

30. MINSAP. Documento elaborado por Cuba para la intervención en la delegación cubana en la 62 Asamblea Mundial de la Salud. Crisis Financiera y Salud. La Habana: MINSAP; 2009.

31. OPS. Agenda de Salud para las Américas 2008-2017. Reunión de Ministros de Salud de la Región. Ciudad de Panamá: OPS; 2007.

32. OMS. Informe Mundial de la salud. La Atención Primaria de Salud: Más necesaria que nunca. Ginebra, Suiza, 2008[sitio en Internet]. [citado 16 Oct 2009]. Disponible en: http://www.who.int/whr/es/index.html

33. Proyecto de Resolución EB124.R8. Atención Primaria de Salud, incluida el fortalecimiento de los sistemas de salud. 62 Asamblea Mundial de la Salud. 20 de mayo de 2009. Ginebra: OMS; 2009.

34. Balaguer JR. Discurso pronunciado en la 62 Asamblea de la OMS. Ginebra, Suiza, 19 mayo 2009 [sitio en Internet]. [citado 16 Oct 2009]. Disponible en: http://www.cubanoal.cu/noticias.html

35. Hadad JL. La protección social como estrategia de enfrentamiento de una crisis económica. Experiencia de Cuba en el sector salud. La Habana: MINSAP; 2009.

36. Pérez V. Experiencia cubana para enfrentar crisis económica. La Habana: Instituto Nacional de Investigaciones Económicas, Universidad de la Habana; 2009.

37. Sistema Nacional de Salud: Políticas, estrategias y Programas. La Habana: MINSAP; 1998.

38. MINSAP. Documento de Trabajo. Proyecciones de la salud pública en Cuba para el 2015. La Habana: MINSAP; 2006.

39. Luna C. Atención primaria de Salud en Cuba. Formación y perfeccionamiento de Recursos Humanos. La Habana: MINSAP; 2009.

40. Castro Ruz R. Discurso del General de Ejército Raúl Castro Ruz, Presidente de los Consejos de Estado y de Ministros en el Tercer Periodo Ordinario de Sesiones de la Asamblea Nacional del Poder Popular. La Habana, Cuba, 1 agosto 2009. Granma digital [sitio en Internet]. [citado 21 Dic 2009]. Disponible en:

http://www.granma.cubaweb.cu

41. MINSAP. Políticas, lineamientos e indicaciones del MINSAP a aplicar en la propuesta de plan económico 2010. La Habana: MINSAP; 2009.

42. MINSAP. Dirección Nacional de Atención Primaria de Salud. La Atención Primaria de Salud en Cuba, su perfeccionamiento. La Habana: MINSAP; 2008.

43. MINSAP. Dirección Nacional de Estadísticas. Anuario estadístico MINSAP 2008. La Habana: MINSAP; 2009.

44. MINREX. Informe de Cuba sobre la Resolución 63/7 de la Asamblea General de las Naciones Unidas. "Necesidad de poner fin al bloqueo económico, comercial y financiero impuesto por Estados Unidos de América contra Cuba". La Habana, Cuba, 
2009 [sitio en Internet]. [citado 23 Oct 2009]. Disponible en: http://www.cubavsbloqueo.cu/Informe2009/3 2.html

45. Osa JA. Condena Mundial a EEUU. (Reseña). Granma. 29 Oct 2009; sección "Internacionales": 8(col.1).

Rodríguez Bruno. Discurso en la Asamblea General de Naciones Unidas. Necesidad de poner fin al bloqueo económico, comercial y financiero impuesto por Estados Unidos contra Cuba. Nueva York. 28 de Octubre 2009. Granma digital [sitio en Internet:]. [citado 29 Oct 2009]. Disponible en:

http://www.radiobayamo.icrt.cu/noticia.php?id=5130

46. MINSAP. Informe anual sobre afectaciones del bloqueo en la salud cubana. Ciudad Habana, Cuba; Junio 2009 [sitio en Internet]. [citado 23 Oct 2009]. Disponible en:

http://www.cubavsbloqueo.cu/Informe2008/informes $\% 20$ de $\% 20$ sectores/BloqueoM INSAP 2008.doc

47. Evans RG, Mckeown T. Meet Fidel Castro: Physicians, Population Health and the Cuban Paradox. Healthcare Policy. 2008;3(4):21-32.

48. World Health Organization. Speeches 2009. Dr. Margaret Chan. Address at Pedro Kouri Institute of Tropical Medicine. WHO. Havana Cuba 26 October 2009 [Sitio en Internet:]. [citado 27 Oct 2009]. Disponible en:

http://www.who.int/about/licensing/rss/es/index.html

Recibido: 5 de marzo de 2010.

Aprobado: 10 de mayo de 2010.

Nestor Marimón Torres. Relaciones Internacionales del Ministerio de Salud Pública. Calle 23 esq. N. EL Vedado 10400. La Habana, Cuba.

E-mail: dirdri@informed.sld.cu, evemart@infomed.sld.cu 